\title{
SOLVING A SPECIFIC THUE-MAHLER EQUATION
}

\author{
N. TZANAKIS AND B. M. M. DE WEGER
}

\begin{abstract}
The diophantine equation $x^{3}-3 x y^{2}-y^{3}= \pm 3^{n_{0}} 17^{n_{1}} 19^{n_{2}}$ is completely solved as follows. First, a large upper bound for the variables is obtained from the theory of linear forms in $p$-adic and real logarithms of algebraic numbers. Then this bound is reduced to a manageable size by $p$-adic and real computational diophantine approximation, based on the $L^{3}$-algorithm. Finally the complete list of solutions is found in a sieving process. The method is in principle applicable to any Thue-Mahler equation, as the authors will show in a forthcoming paper.
\end{abstract}

\section{INTRODUCTION}

Let $f(X, Y) \in \mathbb{Z}[X, Y]$ be a binary form with at least three distinct linear factors over $\mathbb{C}$. Let $p_{1}, \ldots, p_{s}$ be fixed prime numbers. The diophantine equation

$$
f(x, y)= \pm \prod_{i=1}^{s} p_{i}^{n_{i}}
$$

in the variables $x, y \in \mathbb{Z}$ and $n_{1}, \ldots, n_{s} \in \mathbb{Z}_{\geq 0}$ with $(x, y)=1$ is known as a 'Thue-Mahler equation.' It is well known that this equation has only finitely many solutions (Mahler), and that they can, at least in principle, be determined effectively, since an effectively computable upper bound for the variables can be derived from the $p$-adic theory of linear forms in logarithms (Baker, Coates, and Sprindžuk). For the history of the Thue-Mahler equation we refer to [3, Chapter 7].

In this paper we work out a paradigmatic example of determining all the solutions of a specific Thue-Mahler equation. In this way we hope to convince the reader that it is possible to solve any Thue-Mahler equation, not only in principle, but also in practice. We use the (real and $p$-adic) theory of linear forms in logarithms of algebraic numbers, for obtaining explicit but very large upper bounds for the unknowns. Then we reduce these bounds considerably by a combination of real and $p$-adic computational diophantine approximation

Received September 21, 1989.

1980 Mathematics Subject Classification (1985 Revision). Primary 11D25, 11 Y50.

The second author was partially supported by the Netherlands Foundation for Mathematics (SMC) with finanical aid from the Netherlands Organization for the Advancement of Pure Research (ZWO). Thanks are also due to the University of Crete for the hospitality during his visit there in the fall of 1986, when this paper originated. 
techniques based on the $L^{3}$-algorithm for reducing bases of lattices. For a survey of such techniques and examples, see [7], and for an outline of the general method for solving Thue-Mahler equations, see [6]. In a forthcoming publication we intend to give a general and detailed treatment, such as we did for the Thue equation (cf. [4]). Since the present paper is more or less a sequel to [4], we keep the same numbering of the constants $c_{1}, c_{2}, \ldots$.

From now on we concentrate on the following example. Let $f(x, y)=$ $x^{3}-3 x y^{2}-y^{3}$. We study the equation

$$
f(x, y)= \pm 3^{n_{0}} 17^{n_{1}} 19^{n_{2}}
$$

in $x, y \in \mathbb{Z}, n_{0}, n_{1}, n_{2} \in \mathbb{Z}_{\geq 0}$ with $(x, y)=1$. Note that 3,17 , and 19 are the only primes below 20 that occur as prime factors of $f(x, y)$ for $x, y \in \mathbb{Z}$ with $(x, y)=1$. We were motivated to choose this Thue-Mahler equation as a paradigmatic example by the following facts: the cubic field generated by the roots of $f(x, 1)=0$ is totally real, so that there are two fundamental units; this field is a Galois field and has class number 1, which saves us from some trouble; the number of primes $p_{i}$ is larger than one; and there are interestingly large solutions (the only other example of a Thue-Mahler equation that has been treated before by (a variant of) the method of this paper, involved only one fundamental unit and one prime (cf. [1])).

Consider the transformation

$$
\tau(x, y)=(y,-(x+y)) .
$$

It is straightforward to check that

$$
\begin{gathered}
\tau^{2}(x, y)=(-(x+y), x), \quad \tau^{3}=\mathrm{id}, \\
f(x, y)=f(\tau(x, y)) .
\end{gathered}
$$

Thus, every solution of (1) belongs to a class of six solutions:

$$
\left\{ \pm(x, y), \pm \tau(x, y), \pm \tau^{2}(x, y)\right\} .
$$

These six solutions are distinct, and there is just one among them, $(x, y)$ say, with $x>0, y \geq 0$.

\section{THE CUBIC FIELD}

We work in the field $K=\mathbb{Q}(\vartheta)$, where $\vartheta$ is defined by $f(\vartheta, 1)=0 . K$ is a Galois field with class number 1 and discriminant $81=3^{4}$. The conjugates of $\vartheta$ are $\sigma(\vartheta)=2-\vartheta^{2}$ and $\sigma^{2}(\vartheta)=-2-\vartheta+\vartheta^{2}$, where $\sigma$ generates the Galois group.

The mappings $\tau$ and $\sigma$ are related as follows. Put $\tau(x, y)=\left(x_{\tau}, y_{\tau}\right)$. Then, as can easily be checked,

$$
\vartheta \cdot \sigma(x-y \vartheta)=x_{\tau}-y_{\tau} \vartheta .
$$

Note that $\mathscr{O}_{K}=\mathbb{Z}[\vartheta]$ is the ring of integers of $K$, and that a system of fundamental units of $\mathscr{O}_{K}$ is given by $\{\vartheta, \eta\}$ with $\eta=1+\vartheta$. Investigating how 3, 
17 , and 19 factorize into prime ideals of $\mathscr{O}_{K}$, we find that 3 is totally ramified, and that 17 and 19 split completely. In fact, we have:

\begin{tabular}{c|c|c|c}
\hline$\cdot$ & $\sigma(\cdot)$ & $\sigma^{2}(\cdot)$ & $N(\cdot)$ \\
\hline$\vartheta$ & $-\vartheta^{-1} \eta=2-\vartheta^{2}$ & $-\eta^{-1}=-2-\vartheta+\vartheta^{2}$ & 1 \\
$\eta=1+\vartheta$ & $-\vartheta^{-1}=3-\vartheta^{2}$ & $\vartheta \eta^{-1}=-1-\vartheta+\vartheta^{2}$ & -1 \\
$\pi=3-\vartheta$ & $1+\vartheta^{2}$ & $5+\vartheta-\vartheta^{2}$ & 17 \\
$\rho=3+\vartheta$ & $5-\vartheta^{2}$ & $1-\vartheta+\vartheta^{2}$ & 19 \\
$\psi=-1+\vartheta$ & $-\eta \psi=1-\vartheta^{2}$ & $-\vartheta^{-1} \eta \psi=-3-\vartheta+\vartheta^{2}$ & $\vartheta^{-1} \eta^{2} \psi^{3}=3$ \\
\hline
\end{tabular}

\section{FACTORING THE EQUATION IN THE FIELD}

Put $\beta=x-y \vartheta$, where $x, y$ is any solution of (1). Then

$$
N(\beta)= \pm 3^{n_{0}} 17^{n_{1}} 19^{n_{2}} .
$$

Suppose that $\alpha \mid(\beta, \sigma(\beta))$ for a prime $\alpha \in \mathscr{O}_{K}$. Then $\alpha \mid(\beta-\sigma(\beta))=$ $-y(\vartheta-\sigma(\vartheta))$. If $\alpha \mid y$ then, by $y \in \mathbb{Z}$, also $N(\alpha) \mid y$. Also $N(\alpha) \mid N(\beta)=$ $f(x, y)$. Hence $N(\alpha) \mid x$, and this contradicts $(x, y)=1$. So it follows that $\alpha \nmid y$, and thus $\alpha \mid(\vartheta-\sigma(\vartheta))=\vartheta^{-1} \eta^{2} \psi^{2}$. Therefore we may take $\alpha=\psi$. If $\psi^{2} \mid \beta$, then $9=N\left(\psi^{2}\right) \mid N(\beta)=f(x, y)$. By considering $f(x, y)(\bmod 9)$, we find that $9 \mid f(x, y)$ if and only if $3 \mid x$ and $3 \mid y$. By $(x, y)=1$ it follows that $\psi^{2}+\beta$. Hence $(\beta, \sigma(\beta))=(\psi)^{n_{0}}$ for $n_{0} \in\{0,1\}$, and other values for $n_{0}$ in equation (1) are impossible.

In view of the above discussion, equation (1) now yields

$$
\beta=\varepsilon \psi^{n_{0}} \sigma^{i}(\pi)^{n_{1}} \sigma^{j}(\rho)^{n_{2}},
$$

where $\varepsilon \in \mathscr{O}_{K}$ is a unit, and $i, j \in\{0,1,2\}$. We claim that in (3) we may assume without loss of generality that $i=0$, i.e., $\sigma^{i}(\pi)=\pi$. Applying $\sigma$ to (2) yields

$$
-\eta \cdot \sigma^{2}(x-y \vartheta)=x_{\tau^{2}}-y_{\tau^{2}} \vartheta
$$

We apply $\sigma$ once to (3) if $i=2$, and twice if $i=1$. Then (2) or (4) state that we find again an equation of type (3), with $i=0$, with a $\beta$ corresponding to a solution $(x, y)$ that is in the same class of six solutions generated by $\pm \tau$, and with a somewhat different unit $\varepsilon$. This proves our claim.

For $\alpha \in K$ and $h \in\{0,1,2\}$ we will sometimes write $\alpha_{h}$ instead of $\sigma^{h}(\alpha)$. With this convention it suffices to solve

$$
\beta=x-y \vartheta= \pm \psi^{n_{0}} \pi^{n_{1}} \rho_{h}^{n_{2}} \vartheta^{\alpha_{1}} \eta^{\alpha_{2}},
$$


where $n_{0} \in\{0,1\}$ and $h \in\{0,1,2\}$, and where the variables are $x, y \in \mathbb{Z}$, $n_{1}, n_{2} \in \mathbb{Z}_{\geq 0}$, and $a_{1}, a_{2} \in \mathbb{Z}$.

\section{Notation}

For a rational prime $p$ (in our case $p=17$ or 19 ) we denote by $\mathbb{Z}_{p}$ the ring of $p$-adic integers, by $\mathbb{Q}_{p}$ the field of the $p$-adic numbers (the completion of $\mathbb{Q}$ under the $p$-adic metric), and by $\mathbb{C}_{p}$ the completion (under the $p$-adic metric) of the algebraic closure $\overline{\mathbb{Q}}_{p}$ of $\mathbb{Q}_{p}$. In general, if $[\mathbb{Q}(\vartheta): \mathbb{Q}]=n$ and $F(X) \in \mathbb{Q}[X]$ is the minimal polynomial of $\vartheta$ over $\mathbb{Q}$, there are $n$ isomorphic embeddings $\varphi_{i} \quad(i=1, \ldots, n)$ of $\mathbb{Q}(\vartheta)$ into $\mathbb{C}_{p}$. For every $\varphi_{i}$ we have $\varphi_{i}(a)=a$ if $a \in \mathbb{Q}$ and $F\left(\varphi_{i}(\vartheta)\right)=0$. For simplicity in our notation we put $\varphi_{i}(\alpha)=\alpha^{(i)}$ for every $\alpha \in \mathbb{Q}(\vartheta)$. If we work with more than one prime $p$, we will use the same notation $\alpha^{(i)}$, and it will be clear from the context with which prime $p$ we are working. The above remarks remain valid for the infinite prime' $p=\infty$. For this 'prime' the $p$-adic metric is the usual absolute value, and $\mathbb{Q}_{p}=\mathbb{R}, \mathbb{C}_{p}=\mathbb{C}$. Finally, if $\xi \in \mathbb{Q}_{p}$ and $\xi$ has the $p$-adic expansion

$$
\begin{array}{r}
\xi=u_{-m} p^{-m}+\cdots+u_{-1} p^{-1}+u_{0}+u_{1} p+u_{2} p^{2}+\cdots \\
\left(0 \leq u_{j} \leq p-1, m \geq 0\right),
\end{array}
$$

we write $\xi=u_{-m} \cdots u_{-1} \cdot u_{0} u_{1} u_{2} \cdots$. In particular, if $\xi \in \mathbb{Z}_{p}$, we write $\xi=$ $0 . u_{0} u_{1} u_{2} \cdots$.

We make the following convention for numbering the conjugates of $\vartheta$ :

\begin{tabular}{c|c|c|c}
\hline & in $\mathbb{R}$ & in $\mathbb{Q}_{17}$ & in $\mathbb{Q}_{19}$ \\
\hline$\vartheta^{(1)}$ & $1.879385241 \ldots$ & $0.3121414 \ldots$ & $0.163510 \ldots$ \\
$\vartheta^{(2)}$ & $-1.532088886 \ldots$ & $0.101258 \ldots$ & $0.124151 \ldots$ \\
$\vartheta^{(3)}$ & $-0.3472963554 \ldots$ & $0.491310 \ldots$ & $0.1010176 \ldots$ \\
\hline
\end{tabular}

\section{The $S$-Unit EQUATION}

We now return to equation (1), or rather to (5). Consider the three conjugate relations $\sigma^{i}(\beta)=x-y \sigma^{i}(\vartheta)$. Eliminate $x$ and $y$ to obtain

$$
\delta \frac{\sigma^{2}(\beta)}{\sigma(\beta)}-1=\delta^{\prime} \frac{\beta}{\sigma(\beta)},
$$

where

$$
\delta=\frac{\vartheta-\sigma(\vartheta)}{\vartheta-\sigma^{2}(\vartheta)}=\vartheta^{-1} \eta, \quad \delta^{\prime}=\frac{\sigma^{2}(\vartheta)-\sigma(\vartheta)}{\vartheta-\sigma^{2}(\vartheta)}=\vartheta^{-1}
$$


We want to substitute (5) into (6). Put

$$
\begin{array}{ll}
\xi=\frac{\sigma^{2}(\pi)}{\sigma(\pi)}=\frac{75+10 \vartheta-23 \vartheta^{2}}{17}, & \xi^{*}=\frac{\pi}{\sigma(\pi)}=\frac{1}{\sigma^{2}(\xi)}=\frac{52-\vartheta-13 \vartheta^{2}}{17} \\
\chi=\frac{\sigma^{2}(\rho)}{\sigma(\rho)}=\frac{3-4 \vartheta+11 \vartheta^{2}}{19}, & \chi^{*}=\frac{\rho}{\sigma(\rho)}=\frac{1}{\sigma^{2}(\chi)}=\frac{14+13 \vartheta+7 \vartheta^{2}}{19} .
\end{array}
$$

Using our knowledge on the action of $\sigma$ and $\sigma^{2}$ on $\vartheta, \eta$, and $\psi$, we obtain from (6) the following so-called ' $S$-unit equation':

$$
(-1)^{a_{2}} \xi^{n_{1}} \chi_{h}^{n_{2}} \vartheta^{a_{1}^{\prime}} \eta^{a_{2}^{\prime}}-1=(-1)^{a_{1}+a_{2}+n_{0}} \xi^{* n_{1}} \chi_{h}^{* n_{2}} \vartheta^{2 a_{1}+a_{2}-1} \eta^{-a_{1}+a_{2}-n_{0}}
$$

where

$$
a_{1}^{\prime}=a_{1}+2 a_{2}-n_{0}-1, \quad a_{2}^{\prime}=-2 a_{1}-a_{2}+1 .
$$

Note that $\psi$ has disappeared, but $n_{0}$ still occurs.

\section{BOUNDING THE $p$-ADIC ORDERS}

Now we work $\wp$-adically with a conveniently chosen prime ideal $\wp$ over $p$, where $p \in\{17,19\}$. For $p=17$ we take $\wp=\wp_{17}=(\pi)$, and for $p=19$ we take $\wp=\wp_{19}=(\rho)$. Note that for both $p$ 's we have $\operatorname{ord}_{\wp}(\cdot)=\operatorname{ord}_{p}(\cdot)$. From the definition of $\xi^{*}$ and $\chi^{*}$ it is clear that $\operatorname{ord}_{\wp_{17}}\left(\xi^{*}\right)=1, \operatorname{ord}_{\wp_{17}}\left(\chi^{*}\right)=0$, and, since $\vartheta$ and $\eta$ are units in $\mathscr{O}_{K}$, also $\operatorname{ord}_{\wp_{17}}(\vartheta)=\operatorname{ord}_{\wp_{17}}(\eta)=0$. Therefore, (7) immediately implies the relation

$$
n_{1}=\operatorname{ord}_{\wp_{17}}\left((-1)^{a_{2}} \xi^{n_{1}} \chi_{h}^{n_{2}} \vartheta^{a_{1}^{\prime}} \eta^{a_{2}^{\prime}}-1\right) .
$$

When $p=19$, we apply to (7) the automorphism $\sigma^{-h}$ and get

$$
(-1)^{a_{2}} \xi_{-h}^{n_{1}} \chi^{n_{2}} \vartheta_{-h}^{a_{1}^{\prime}} \eta_{-h}^{a_{2}^{\prime}}-1=(-1)^{a_{1}+a_{2}+n_{0}} \xi_{-h}^{* n_{1}} \chi^{* n_{2}} \vartheta_{-h}^{2 a_{1}+a_{2}-1} \eta_{-h}^{-a_{1}+a_{2}-n_{0}} .
$$

Note that $\operatorname{ord}_{\wp_{19}}\left(\chi^{*}\right)=1$, while all other factors in the right-hand side have $\wp_{19}$-adic order equal to zero. Therefore,

$$
n_{2}=\operatorname{ord}_{\wp_{19}}\left((-1)^{a_{2}} \xi_{-h}^{n_{1}} \chi^{n_{2}} \vartheta_{-h}^{a_{1}^{\prime}} \eta_{-h}^{a_{2}^{\prime}}-1\right)
$$

We put

$$
N=\max \left\{n_{1}, n_{2}\right\}, \quad A=\max \left\{\left|a_{1}^{\prime}\right|,\left|a_{2}^{\prime}\right|\right\}, \quad H=\max \{A, N\} .
$$

Then by

$$
a_{1}=\frac{1}{3}\left(-a_{1}^{\prime}-2 a_{2}^{\prime}-n_{0}+1\right), \quad a_{2}=\frac{1}{3}\left(2 a_{1}^{\prime}+a_{2}^{\prime}+2 n_{0}+1\right),
$$

it follows that

$$
\left|a_{1}\right| \leq A, \quad\left|a_{2}\right| \leq A+1,
$$

with $\left|a_{2}\right|=A+1$ only if $a_{1}^{\prime}=a_{2}^{\prime}=A$ and $n_{0}=1$. We now can apply K. R. Yu's theorem (cf. [9]) to the right-hand sides of (8) and (9) in order to find upper bounds for them, which are of the form $c_{13}\left(\log H+c_{14}\right)$ (for the 
precise formulation of Yu's theorem see the Appendix). We have calculated (see the Appendix) that in both cases we can take

$$
c_{13}=6.190047 \cdot 10^{24}, \quad c_{14}=4.28 \text {. }
$$

Thus, from (8) and (9) it follows that

$$
N \leq c_{13}\left(\log H+c_{14}\right) \text {. }
$$

\section{UPPER BOUNDS FOR ALL THE VARIABLES}

Inequality (10) will play an important role in computing an upper bound for $H$, a task which we undertake in this section. First we need the following lemma.

Lemma 1. For the unit $\varepsilon=\vartheta^{a_{1}^{\prime}} \eta^{a_{2}^{\prime}}$ (appearing in the right-hand side of (7)) there exists an index $k \in\{2,3\}$ such that

$$
\text { either }\left|\varepsilon^{(k)}\right| \geq e^{c_{15} A} \quad \text { or }\left|\varepsilon^{(k)}\right| \leq e^{-c_{15} A},
$$

where, as before, $A=\max \left\{\left|a_{1}^{\prime}\right|,\left|a_{2}^{\prime}\right|\right\}$, and $c_{15}=0.572215$.

Proof. We have

from which we derive

$$
\left(\begin{array}{l}
\log \left|\varepsilon^{(2)}\right| \\
\log \left|\varepsilon^{(3)}\right|
\end{array}\right)=\left(\begin{array}{ll}
\log \left|\vartheta^{(2)}\right| & \log \left|\eta^{(2)}\right| \\
\log \left|\vartheta^{(3)}\right| & \log \left|\eta^{(3)}\right|
\end{array}\right)\left(\begin{array}{c}
a_{1}^{\prime} \\
a_{2}^{\prime}
\end{array}\right)
$$

$$
\left(\begin{array}{l}
a_{1}^{\prime} \\
a_{2}^{\prime}
\end{array}\right)=\frac{-1}{R}\left(\begin{array}{cc}
\log \left|\eta^{(3)}\right| & -\log \left|\eta^{(2)}\right| \\
-\log \left|\vartheta^{(3)}\right| & \log \left|\vartheta^{(2)}\right|
\end{array}\right)\left(\begin{array}{l}
\log \left|\varepsilon^{(2)}\right| \\
\log \left|\varepsilon^{(3)}\right|
\end{array}\right)
$$

where $R \quad \approx 0.849287450)$ is the regulator of the field $K$. Computing approximately the elements of the matrix in the right-hand side, we obtain

$$
\begin{aligned}
& \left|a_{1}^{\prime}\right| \leq 0.502341215|\log | \varepsilon^{(2)}\left\|+0.742910686|\log | \varepsilon^{(3)}\right\|, \\
& \left|a_{2}^{\prime}\right| \leq 1.245251901|\log | \varepsilon^{(2)}\left\|+0.502341215|\log | \varepsilon^{(3)}\right\| .
\end{aligned}
$$

Let $k \in\{2,3\}$ be such that $|\log | \varepsilon^{(k)} \|=\max \left\{|\log | \varepsilon^{(2)}\left\|,|\log | \varepsilon^{(3)}\right\|\right\}$. From the above inequalities it follows that

$$
A \leq 1.747593116|\log | \varepsilon^{(k)} \|,
$$

i.e.,

$$
|\log | \varepsilon^{(k)}|| \geq c_{15} A,
$$

from which the result follows.

We now take a positive constant $c_{16}<\frac{1}{2} c_{15}$ (e.g., $c_{16}=0.085$ ), and for our further study we distinguish three cases. In the first two of them, $k$ will be the index defined by Lemma 1 .

Case 1. $\min _{1 \leq i \leq 3}\left|\beta^{(i)}\right|>e^{-c_{16} A}$ and $\left|\varepsilon^{(k)}\right| \geq e^{c_{15} A}$. We have $\left|\beta^{(k)}\right| \prod_{i \neq k}\left|\beta^{(i)}\right|=$ $3^{n_{0}} 17^{n_{1}} 19^{n_{2}}$ and then, in view of the first inequality above,

$$
\left|\beta^{(k)}\right|<3^{n_{0}} 17^{n_{1}} 19^{n_{2}} e^{2 c_{16} A} .
$$


Then

$$
\begin{aligned}
e^{c_{15} A} & \leq\left|\varepsilon^{(k)}\right|=\frac{\left|\beta^{(k)}\right|}{\left|\psi^{(k)}\right|^{n_{0}}\left|\pi^{(k)}\right|^{n_{1}}\left|\rho_{h}^{(k)}\right|^{n_{2}}} \leq\left|\frac{3}{\psi^{(k)}}\right|^{n_{0}}\left|\frac{17}{\pi^{(k)}}\right|^{n_{1}}\left|\frac{19}{\rho_{h}^{(k)}}\right|^{n_{2}} e^{2 c_{16} A} \\
& <3.2266816 \cdot 5.0787257^{n_{1}} \cdot 12.94356302^{n_{2}} e^{2 c_{16} A} \\
& <e^{c_{18}+c_{17} N+2 c_{16} A},
\end{aligned}
$$

where $c_{17}=4.185659$ and $c_{18}=1.1715$. It follows that

$$
\left(c_{15}-2 c_{16}\right) A<c_{18}+c_{17} N .
$$

Case 2. $\min _{1 \leq i \leq 3}\left|\beta^{(i)}\right|>e^{-c_{16} A}$ and $\left|\varepsilon^{(k)}\right| \leq e^{-c_{15} A}$. Now

$$
\begin{aligned}
e^{-c_{15} A} & \geq\left|\varepsilon^{(k)}\right|=\frac{\left|\beta^{(k)}\right|}{\left|\psi^{(k)}\right|^{n_{0}}\left|\pi^{(k)}\right|^{n_{1}}\left|\rho_{h}^{(k)}\right|^{n_{2}}}>\frac{e^{-c_{16} A}}{\left.|\psi| \pi\right|^{n_{1}}|\rho|^{n_{2}}} \\
& \geq \frac{e^{-c_{16} A}}{\mid \psi\left((\pi|\rho| \rho)^{N}\right.}>e^{-\left(c_{18}^{\prime}+c_{17}^{\prime} N+c_{16} A\right)},
\end{aligned}
$$

where $c_{17}^{\prime}=3.0962022$ and $c_{18}^{\prime}=0.93$. Then

$$
\left(c_{15}-c_{16}\right) A<c_{18}^{\prime}+c_{17}^{\prime} N \text {. }
$$

Before we proceed with the third case, we show how in Cases 1 and 2 an upper bound for $H$ follows. Combining (12) with (10) we get for Case 1:

$$
A<c_{19}+c_{20} \log H \text {, }
$$

where

$$
c_{19}=\frac{c_{18}+c_{13} c_{14} c_{17}}{c_{15}-2 c_{16}}, \quad c_{20}=\frac{c_{13} c_{17}}{c_{15}-2 c_{16}} .
$$

On the other hand, by (13) and (10), on noting that $c_{17}^{\prime}<c_{17}$ and $c_{18}^{\prime}<c_{18}$, we get the same for Case 2. Finally, (10) implies that $N<c_{19}+c_{20} \log H$, and therefore in both Case 1 and Case 2 we have by the definition of $H$,

$$
H<c_{19}+c_{20} \log H,
$$

from which we easily get the following upper bound for $H$ :

$$
H<4.38 \cdot 10^{27} \text {. }
$$

Case 3. $\min _{1 \leq i \leq 3}\left|\beta^{(i)}\right| \leq e^{-c_{16} A}$. We put $\left|\beta^{\left(i_{0}\right)}\right|=\min _{1 \leq i \leq 3}\left|\beta^{(i)}\right|$. Then for any $j \neq i_{0} \quad(j \in\{1,2,3\})$ we have $|y|\left|\vartheta^{(j)}-\vartheta^{\left(i_{0}\right)}\right|=\left|\beta^{(j)}-\beta^{\left(i_{0}\right)}\right| \leq 2\left|\beta^{(j)}\right|$, from which

$$
\left|\beta^{(j)}\right| \geq \frac{1}{2} \min _{l \neq i}\left|\vartheta^{(l)}-\vartheta^{(i)}\right| .
$$

Put $(\sigma(\beta))^{\left(i_{0}\right)}=\beta^{(j)}$ and $\left(\sigma^{2}(\beta)\right)^{\left(i_{0}\right)}=\beta^{(k)}$ for $i_{0} \neq j \neq k \neq i_{0}$. We have $\left|\delta^{\prime\left(i_{0}\right)} \beta^{\left(i_{0}\right)} / \beta^{(j)}\right|<c_{21} e^{-c_{16} A}$, where

$$
c_{21}=1.7057371>\frac{1}{2} \min _{l \neq i}\left|\vartheta^{(l)}-\vartheta^{(i)}\right| \cdot \mid \delta^{\prime} .
$$


Then, in view of (6),

$$
\left|\delta^{\left(i_{0}\right)} \frac{\beta^{(k)}}{\beta^{(j)}}-1\right|<c_{21} e^{-c_{16} H}<0.72542132,
$$

provided that $H \geq 3$. In particular, $\delta^{\left(i_{0}\right)} \beta^{(k)} / \beta^{(j)}>0$, and therefore

$$
\delta^{\left(i_{0}\right)} \frac{\beta^{(k)}}{\beta^{(j)}}=\left|\delta^{\left(i_{0}\right)} \frac{\beta^{(k)}}{\beta^{(j)}}\right|=\left|\left\{\delta \frac{\sigma^{2}(\beta)}{\sigma(\beta)}\right\}^{\left(i_{0}\right)}\right|=\left|\xi^{\left(i_{0}\right)}\right|^{n_{1}}\left|\chi_{h}^{\left(i_{0}\right)}\right|^{n_{2}}\left|\vartheta^{\left(i_{0}\right)}\right|^{a_{1}^{\prime}}\left|\eta^{\left(i_{0}\right)}\right|^{a_{2}^{\prime}}=e^{\Lambda_{0}},
$$

where

$$
\Lambda_{0}=n_{1} \log \left|\xi^{\left(i_{0}\right)}\right|+n_{2} \log \left|\chi_{h}^{\left(i_{0}\right)}\right|+a_{1}^{\prime} \log \left|\vartheta^{\left(i_{0}\right)}\right|+a_{2}^{\prime} \log \left|\eta^{\left(i_{0}\right)}\right|
$$

It is clear from (6) that $\Lambda_{0} \neq 0$. Also, from (15) we have $\left|e^{\Lambda_{0}}-1\right|<$ 0.72542132 , from which we easily see that $\left|\Lambda_{0}\right|<1.782\left|e^{\Lambda_{0}}-1\right|$ (namely, if $\left|e^{x}-1\right|<\delta<1$, then $\left.|x|<-(\log (1-\delta) / \delta)\left|e^{x}-1\right|\right)$. Thus, we finally get

$$
0<\left|\Lambda_{0}\right|<1.782 c_{21} e^{-c_{16} H} .
$$

By Waldschmidt's theorem (cf. [5]) positive constants $c_{7}$ and $c_{8}$ can be computed such that

$$
\left|\Lambda_{0}\right|>e^{-c_{7}\left(\log H+c_{8}\right)} .
$$

Using the version of this theorem as it is formulated in Appendix II of [4], we have computed the following values:

$$
c_{7}=2.6467 \cdot 10^{29}, \quad c_{8}=2.442325 \text {. }
$$

On combining (17) with (18) we get

$$
H<\frac{c_{7} c_{8}+\log \left(1.782 c_{21}\right)}{c_{16}}+\frac{c_{7}}{c_{16}} \log H
$$

which implies the following upper bound for $H$ in Case 3:

$$
H<5.76 \cdot 10^{32} \text {. }
$$

Comparing this upper bound with that for Cases 1 and 2 (cf. (14)), we see that in any case (19) is true.

\section{REAL AND $p$-ADIC LINEAR FORMS IN LOGARITHMS}

In the forthcoming sections we show how the upper bound for $H$ given in (19) can be reduced. First we transform equations (8) and (9) into equations involving linear forms in $p$-adic logarithms of numbers that are algebraic over $\mathbb{Q}_{p}$, with $p=17$ and 19 , respectively. We need a simple general lemma.

Lemma 2. If $z \in \mathbb{Z}_{p}$, $\operatorname{ord}_{p}(z-1) \geq 1$, and $k \in \mathbb{N}$ with $p \nmid k$, then

$$
\operatorname{ord}_{p}\left(z^{k}-1\right)=\operatorname{ord}_{p}(z-1) \text {. }
$$


The proof is an easy exercise, which we leave to the reader. We will also make use of the following

Lemma 3. Let $z_{1}, \ldots, z_{m} \in \mathbb{C}_{p}$ with $\operatorname{ord}_{p}\left(z_{i}-1\right)>1 /(p-1)(1 \leq i \leq m)$, and $n_{1}, \ldots, n_{m} \in \mathbb{C}_{p}$ with ord $_{p}\left(n_{i}\right) \geq 0 \quad(1 \leq i \leq m)$. Then the p-adic logarithms $\log _{p} z_{i}$ are defined for $1 \leq i \leq m$, and

$$
\operatorname{ord}_{p}\left(z_{1}^{n_{1}} \cdots z_{m}^{n_{m}}-1\right)=\operatorname{ord}_{p}\left(n_{1} \log _{p} z_{1}+\cdots+n_{m} \log _{p} z_{m}\right) \text {. }
$$

For a proof of this lemma, see [8, equation (1.2)].

Now we put

$$
\Lambda_{j}=n_{1} \lambda_{j 1}+n_{2} \lambda_{j 2}+a_{1}^{\prime} \mu_{j 1}+a_{2}^{\prime} \mu_{j 2} \quad(j=1,2,0),
$$

where

$$
\begin{array}{ll}
\lambda_{11}=\log _{17}\left(\xi^{(1)^{16}}\right), & \lambda_{12}=\log _{17}\left(\chi_{h}^{(1)^{16}}\right), \\
\mu_{11}=\log _{17}\left(\vartheta^{(1)^{16}}\right), & \mu_{12}=\log _{17}\left(\eta^{(1)^{16}}\right),
\end{array}
$$

all in $\mathbb{Q}_{17}$,

$$
\begin{array}{ll}
\lambda_{21}=\log _{19}\left(\xi_{-h}^{(1)^{18}}\right), & \lambda_{22}=\log _{19}\left(\chi^{(1)^{18}}\right), \\
\mu_{21}=\log _{19}\left(\vartheta_{-h}^{(1)^{18}}\right), & \mu_{22}=\log _{19}\left(\eta_{-h}^{(1)^{18}}\right),
\end{array}
$$

all in $\mathbb{Q}_{19}$, and

$$
\lambda_{01}=\log \left|\xi^{\left(i_{0}\right)}\right|, \quad \lambda_{02}=\log \left|\chi_{h}^{\left(i_{0}\right)}\right|, \quad \mu_{01}=\log \left|\vartheta^{\left(i_{0}\right)}\right|, \quad \mu_{02}=\log \left|\eta^{\left(i_{0}\right)}\right|,
$$

all in $\mathbb{R}$.

We now go back to (8) and apply Lemma 2 to

$$
z=(-1)^{a_{2}} \xi^{(1)^{n_{1}}} \chi_{h}^{(1)^{n_{2}}} \vartheta^{(1)^{a_{1}^{\prime}}} \eta^{(1)^{a_{2}^{\prime}}} \in \mathbb{Q}_{17} .
$$

Since $\operatorname{ord}_{\wp_{17}}(\cdot)=\operatorname{ord}_{17}(\cdot)$ and $\operatorname{ord}_{17}\left(\alpha^{16}-1\right) \geq 1$ for any $\alpha \in K$ with $\operatorname{ord}_{\pi}(\alpha)=$ 0 , it follows from the above two lemmas that

$$
n_{1}=\operatorname{ord}_{17}\left(\Lambda_{1}\right) \text {. }
$$

Analogously, working in $\mathbb{Q}_{19}$, we get from (9)

$$
n_{2}=\operatorname{ord}_{19}\left(\Lambda_{2}\right) \text {. }
$$

Summarizing, we now have the following situation:

- first

$$
\begin{gathered}
n_{1}, n_{2} \in \mathbb{Z}_{\geq 0}, \quad N=\max \left\{n_{1}, n_{2}\right\}, \\
a_{1}^{\prime}, a_{2}^{\prime} \in \mathbb{Z}, \quad A=\max \left\{\left|a_{1}^{\prime}\right|,\left|a_{2}^{\prime}\right|\right\}, \\
H=\max \{A, N\}<K_{0}=5.76 \cdot 10^{32}
\end{gathered}
$$

(cf. (19)); 
- either

$$
A<K_{1}+K_{2} N, \quad \text { where } K_{1}=2.913 \text { and } K_{2}=10.4066
$$

(cf. (12) and (13)),

or

$$
\begin{aligned}
0<\left|\Lambda_{0}\right|<K_{3} e^{-K_{4} H}, & \text { where } K_{3}=3.04>1.782 \cdot c_{22} \\
& \text { and } K_{4}=0.085=c_{16}
\end{aligned}
$$

(cf. (17));

- and finally

$$
\operatorname{ord}_{17}\left(\Lambda_{1}\right)=n_{1} \quad \text { and } \quad \operatorname{ord}_{19}\left(\Lambda_{2}\right)=n_{2} .
$$

\section{THE $p$-ADIC REDUCTION STEP}

We start working in $\mathbb{Q}_{17}$. An easy computation shows that $\operatorname{ord}_{17}\left(\lambda_{1 j}\right)=1$ and $\operatorname{ord}_{17}\left(\mu_{1 j}\right)=1 \quad(j=1,2)$. We write

$$
-\frac{\lambda_{11}}{\lambda_{12}}=\beta_{1} \quad \text { and } \quad-\frac{\mu_{1 j}}{\lambda_{12}}=\alpha_{j} \quad(j=1,2),
$$

so that $\beta_{1}, \alpha_{1}, \alpha_{2} \in \mathbb{Z}_{17}$.

We choose a positive integer $m$ such that $17^{m}$ is of the size of $K_{0}^{4}$, and $m$ should be large enough. We denote by $\beta_{1}^{(m)}$ and $\alpha_{j}^{(m)}$ the unique rational integers in the interval $\left[0,17^{m}-1\right]$ which are congruent $\left(\bmod 17^{m}\right)$ to $\beta_{1}$ and $\alpha_{j}$, respectively $(j=1,2)$. Next, we consider the lattice $\Gamma_{m} \subset \mathbb{Z}^{4}$ generated by the column vectors of the matrix

$$
\mathscr{A}_{m}=\left(\begin{array}{cccc}
1 & 0 & 0 & 0 \\
0 & 1 & 0 & 0 \\
0 & 0 & 1 & 0 \\
\alpha_{1}^{(m)} & \alpha_{2}^{(m)} & \beta_{1}^{(m)} & 17^{m}
\end{array}\right) .
$$

Using the $L^{3}$-algorithm (cf. $[2 ; 7, \S 3.5]$ for an integer version), we computed a reduced basis $\left\{\mathbf{b}_{1}, \ldots, \mathbf{b}_{4}\right\}$ of $\Gamma_{m}$. Then every nonzero vector of $\Gamma_{m}$ has a length which is greater than or equal to $2^{-3 / 2}\left|\mathbf{b}_{i}\right|$ (cf. [2, Proposition (1.11)]), and we expect that this be of size $\operatorname{det}\left(\Gamma_{m}\right)^{1 / 4}=\operatorname{det}\left(\mathscr{A}_{m}\right)^{1 / 4}=17^{m / 4}$, which is of the size of $K_{0}$.

Proposition 4. If $\left|\mathbf{b}_{1}\right|>2^{5 / 2} K_{0}$, then $n_{1} \leq m$.

Proof. Suppose that

$$
n_{1} \geq m+1 \text {. }
$$

We claim that

$$
\left(\begin{array}{l}
a_{1}^{\prime} \\
a_{2}^{\prime} \\
n_{1} \\
n_{2}
\end{array}\right) \in \Gamma_{m}
$$


Indeed,

where

$$
\left(\begin{array}{l}
a_{1}^{\prime} \\
a_{2}^{\prime} \\
n_{1} \\
n_{2}
\end{array}\right)=\mathscr{A}_{m}\left(\begin{array}{l}
a_{1}^{\prime} \\
a_{2}^{\prime} \\
n_{1} \\
\lambda
\end{array}\right)
$$

$$
\lambda=\frac{-a_{1}^{\prime} \alpha_{1}^{(m)}-a_{2}^{\prime} \alpha_{2}^{(m)}-n_{1} \beta_{1}^{(m)}+n_{2}}{17^{m}} .
$$

Then it suffices to show that $\lambda \in \mathbb{Z}$, or, equivalently, that

$$
\operatorname{ord}_{17}\left(-a_{1}^{\prime} \alpha_{1}^{(m)}-a_{2}^{\prime} \alpha_{2}^{(m)}-n_{1} \beta_{1}^{(m)}+n_{2}\right) \geq m \text {. }
$$

By hypothesis,

$$
\operatorname{ord}_{17}\left(\alpha_{j}^{(m)}-\alpha_{j}\right) \geq m \quad(j=1,2) \quad \text { and } \quad \operatorname{ord}_{17}\left(\beta_{1}^{(m)}-\beta_{1}\right) \geq m \text {. }
$$

Therefore, in order that $(28)$ be true, it suffices that

$$
\operatorname{ord}_{17}\left(-a_{1}^{\prime} \alpha_{1}-a_{2}^{\prime} \alpha_{2}-n_{1} \beta_{1}+n_{2}\right) \geq m \text {. }
$$

Note that $-a_{1}^{\prime} \alpha_{1}-a_{2}^{\prime} \alpha_{2}-n_{1} \beta_{1}+n_{2}=\Lambda_{1} / \lambda_{12}$, which means that (29) is equivalent to $\operatorname{ord}_{17}\left(\Lambda_{1}\right) \geq m+\operatorname{ord}_{17}\left(\lambda_{12}\right)=m+1$. But this is true, since in view of (26) and (25) $\operatorname{ord}_{17}\left(\Lambda_{1}\right)=n_{1} \geq m+1$.

Now, (27) is proved, therefore we must have

$$
a_{1}^{\prime 2}+a_{2}^{\prime 2}+n_{1}^{2}+n_{2}^{2} \geq 2^{-3}\left|\mathbf{b}_{1}\right|^{2}
$$

and a fortiori $4 K_{0}^{2} \geq 2^{-3}\left|\mathbf{b}_{1}\right|^{2}$, i.e., $\left|\mathbf{b}_{1}\right| \leq 2^{5 / 2} K_{0}$, which contradicts the assumption of the proposition.

In our application of the above proposition we have chosen $m=110$ or 111 , so that $17^{m}$ is somewhat larger than $K_{0}^{4}$, and we computed a reduced basis for the lattice $\Gamma_{m}$ (there are three possibilities for $\Gamma_{m}$, corresponding to $h=0,1,2$; cf. $(20))$. Then we checked that $\left|\mathbf{b}_{1}\right|>2^{5 / 2} K_{0}$, from which we concluded that $n_{1} \leq 111$.

In an analogous way we worked with the prime $p=19$ in place of $p=17$, and we concluded that $n_{2} \leq 108$. Thus we have found a new, small upper bound $N_{0}=111$ for $N=\max \left\{n_{1}, n_{2}\right\}$, instead of the large bound $K_{0}=$ $5.76 \cdot 10^{32}$. This completes the ' $p$-adic reduction step.'

10. THE REAL REDUCTION STEP

Now, in view of (23) and (24) we have either

$$
A<K_{1}+K_{2} N_{0}<1159
$$

or

$$
0<\left|\Lambda_{0}\right|<K_{3} e^{-K_{4} H}
$$

If the second alternative holds, then we apply the 'real reduction step,' as explained below. Note that there are nine possibilities for $\Lambda_{0}$, corresponding to the nine possibilities for $\left(i_{0}, h\right) \in\{1,2,3\} \times\{0,1,2\}$. 
We choose a 'big' constant $C$ of the size of $K_{0}^{2} N_{0}^{2}$ (in our application we took $C=10^{75}$ or $10^{76}$ ), and we put

$$
\beta_{j}=\left[C \lambda_{0 j}\right], \quad \alpha_{j}=\left[C \mu_{0 j}\right] \quad(j=1,2) .
$$

Here, $[x]=\lfloor x\rfloor$ if $x \geq 0$, and $[x]=\lceil x\rceil$ if $x<0$. Next, we consider the lattice $\Gamma \subset \mathbb{Z}^{4}$ generated by the column vectors of the matrix

$$
\mathscr{A}=\left(\begin{array}{cccc}
{\left[K_{0} / N_{0}\right]} & 0 & 0 & 0 \\
0 & {\left[K_{0} / N_{0}\right]} & 0 & 0 \\
0 & 0 & 1 & 0 \\
\beta_{1} & \beta_{2} & \alpha_{1} & \alpha_{2}
\end{array}\right),
$$

and we find by the $L^{3}$-algorithm a reduced basis $\left\{\mathbf{b}_{1}, \ldots, \mathbf{b}_{4}\right\}$. Then every nonzero vector of $\Gamma$ has a length greater than or equal to $2^{-3 / 2}\left|\mathbf{b}_{1}\right|$, and one expects that this be of the size of $\operatorname{det}(\Gamma)^{1 / 4}=\operatorname{det}(\mathscr{A})^{1 / 4} \approx K_{0}^{1 / 2} N_{0}^{-1 / 2} C^{1 / 4}$, which is of the size of $K_{0}$.

Proposition 5. If $\left|\mathbf{b}_{1}\right|>2^{3 / 2} \sqrt{4\left(K_{0}+N_{0}\right)^{2}+3 K_{0}^{2}}$, then

$$
H<\frac{1}{K_{4}}\left\{\log K_{3}+\log C-\log \left(\sqrt{2^{-3}\left|\mathbf{b}_{1}\right|^{2}-3 K_{0}^{2}}-2\left(K_{0}+N_{0}\right)\right)\right\} .
$$

Proof. Consider the lattice point

$$
\mathbf{x}=\mathscr{A}\left(\begin{array}{c}
n_{1} \\
n_{2} \\
a_{1}^{\prime} \\
a_{2}^{\prime}
\end{array}\right)=\left(\begin{array}{c}
{\left[K_{0} / N_{0}\right] n_{1}} \\
{\left[K_{0} / N_{0}\right] n_{2}} \\
a_{1}^{\prime} \\
\lambda
\end{array}\right) \in \Gamma,
$$

where $\lambda=n_{1} \beta_{1}+n_{2} \beta_{2}+a_{1}^{\prime} \alpha_{1}+a_{2}^{\prime} \alpha_{2}$. It is obvious that $\left|\lambda-C \Lambda_{0}\right|<2 N_{0}+2 K_{0}$, and therefore, also in view of (24),

$$
|\lambda|<C K_{3} e^{-K_{4} H}+2\left(N_{0}+K_{0}\right) .
$$

On the other hand, we must have $|\mathbf{x}| \geq 2^{-3 / 2}\left|\mathbf{b}_{1}\right|$, which is equivalent to

$$
\left[K_{0} / N_{0}\right]^{2}\left(n_{1}^{2}+n_{2}^{2}\right)+a_{1}^{\prime 2}+\lambda^{2} \geq 2^{-3}\left|\mathbf{b}_{1}\right|^{2} .
$$

Since $n_{1}^{2}, n_{2}^{2} \leq N_{0}^{2}$ and $\left|a_{1}^{\prime}\right| \leq K_{0}$, it then follows that $3 K_{0}^{2}+\lambda^{2} \geq 2^{-3}\left|\mathbf{b}_{1}\right|^{2}$, i.e.,

$$
|\lambda|>\sqrt{2^{-3}\left|\mathbf{b}_{1}\right|^{2}-3 K_{0}^{2}}
$$

(note that by hypothesis the argument of the square root is positive). Combine now the last inequality with (31) to get

$$
C K_{3} e^{-K_{4} H}>\sqrt{2^{-3}\left|\mathbf{b}_{1}\right|^{2}-3 K_{0}^{2}}-2\left(N_{0}+K_{0}\right),
$$

and the right-hand side is positive by hypothesis. Taking logarithms, we obtain the desired upper bound for $H$. 
Remark. In practice we estimate the reduced upper bound for $H$ as follows: let $\delta_{1} \in \mathbb{R}$ satisfy $\delta_{1}<\left|\mathbf{b}_{1}\right| / K_{0}$. Then, if the hypothesis of Proposition 5 is true, we have

$$
H<\frac{1}{K_{4}}\left(\log K_{3}+\log C-\log K_{0}-\log \delta_{2}\right),
$$

where $\delta_{2}<\sqrt{\delta_{1}^{2} / 8-3}-2\left(1+N_{0} / K_{0}\right)$.

In our application with the chosen values for $C$, the hypothesis of Proposition 5 was checked to be true, and this implied $H \leq 1169$. Compare with $N \leq 111$ and (30) to get the new reduced upper bound $H \leq 1169$. This is extremely small indeed, compared to the initial upper bound (cf. (22)), but it is still quite large for practical application. Therefore, we applied once more the whole reduction process as described above, but now with $K_{0}=1169$ and in the $p$-adic reduction step $m=14$ for both $p=17$ and $p=19$. The $p$-adic reduction step gave

$$
N \leq 14
$$

and either (23) holds, in which case $A<K_{1}+14 K_{2}<149$, or (24) holds, in which case we can apply the real reduction step with $C=10^{13}$ or $10^{14}$, which yielded $H \leq 296$. Thus, $\max \left\{\left|a_{1}^{\prime}\right|,\left|a_{2}^{\prime}\right|\right\} \leq 296$, and consequently

$$
\max \left\{\left|a_{1}\right|,\left|a_{2}\right|\right\} \leq 297 \text {. }
$$

Numerical details of all our computations can be obtained from the authors on request.

\section{THE FINAL SIEVE}

It remains to check for which values $\left(n_{0}, n_{1}, n_{2}, a_{1}, a_{2}\right)$ satisfying (32) and (33) the coefficient of $\vartheta^{2}$ in the right-hand side of (5) is 0 . We have worked as follows. We have chosen a 'large' number such that the orders of $\vartheta, \eta, \pi$, and $\rho$ modulo this number are 'small.' Such a number is $2701=37.73$, and the orders of $\vartheta, \eta, \pi$, and $\rho(\bmod 2701)$ are all equal to 72 . Fix $n_{0} \in\{0,1\}$ and $h \in\{0,1,2\}$ in (5), and check (by computer, of course) for which quadruples

$$
\left(n_{1}, n_{2}, a_{1}, a_{2}\right) \in\{0, \ldots, 14\}^{2} \times\{0, \ldots, 71\}^{2}
$$

the coefficient of $\vartheta^{2}$ in the right-hand side of $(5)$ is $0(\bmod 2701)$. The number of such quadruples turned out to be between 450 and 500 (depending on $n_{0}$ and $h$ ). Now, in the range determined by (32) and (33) we have only about $4 \cdot 10^{4}$ quadruples to check, instead of the initial $15^{2}(2 \cdot 297+1)^{2} \approx 7.96 \cdot 10^{7}$. Choose a prime $p \neq 37,73$, and of the so selected quadruples keep only those for which the coefficient of $\vartheta^{2}$ in the right-hand side of $(5)$ is $0(\bmod p)$. We did so for $p=601$ and $p=541$ (chosen at random), and were left with about 14 quadruples (the exact number depending on $n_{0}$ and $h$ ), which then could be checked further by straightforward computations.

Note that this sieving process has to be applied six times: for $n_{0}=0,1$ and for $h=0,1,2$. 


\section{CONCLUSION: THE COMPLETE LIST OF SOLUTIONS}

Now we have a complete proof of the following theorem, listing all the solutions of equation (1).

Theorem A. The Thue-Mahler equation

$$
x^{3}-3 x y^{2}-y^{3}= \pm 3^{n_{0}} 17^{n_{1}} 19^{n_{2}}
$$

in $x, y \in \mathbb{Z}$ and $n_{0}, n_{1}, n_{2} \in \mathbb{Z}_{\geq 0}$ with $(x, y)=1$ has exactly the 156 solutions presented in Table 1 .

\section{TABLE 1}

The solutions of equation (1)

\begin{tabular}{|c|c|c|c|c|c|}
\hline$n_{0}$ & $n_{1}$ & $n_{2}$ & & $\pm(x, y)$ & \\
\hline 0 & 0 & 0 & $(1,0)$ & $(0,-1)$ & $(1,-1)$ \\
\hline 0 & 0 & 0 & $(2,1)$ & $(1,-3)$ & $(3,-2)$ \\
\hline 0 & 0 & 1 & $(1,2)$ & $(2,-3)$ & $(3,-1)$ \\
\hline 0 & 0 & 2 & $(4,5)$ & $(5,-9)$ & $(9,-4)$ \\
\hline 0 & 1 & 0 & $(3,1)$ & $(1,-4)$ & $(4,-3)$ \\
\hline 0 & 1 & 0 & $(3,2)$ & $(2,-5)$ & $(5,-3)$ \\
\hline 0 & 1 & 0 & $(15,8)$ & $(8,-23)$ & $(23,-15)$ \\
\hline 0 & 1 & 1 & $(1,6)$ & $(6,-7)$ & $(7,-1)$ \\
\hline 0 & 1 & 1 & $(3,5)$ & $(5,-8)$ & $(8,-3)$ \\
\hline 0 & 1 & 1 & $(28,15)$ & $(15,-43)$ & $(43,-28)$ \\
\hline 0 & 2 & 1 & $(59,31)$ & $(31,-90)$ & $(90,-59)$ \\
\hline 0 & 2 & 1 & $(31,15)$ & $(15,-46)$ & $(46,-31)$ \\
\hline 0 & 2 & 1 & $(18,13)$ & $(13,-31)$ & $(31,-18)$ \\
\hline 0 & 2 & 2 & $(206,109)$ & $(109,-315)$ & $(315,-206)$ \\
\hline 0 & 2 & 5 & $(896,37)$ & $(37,-933)$ & $(933,-896)$ \\
\hline 0 & 3 & 1 & $(97,54)$ & $(54,-151)$ & $(151,-97)$ \\
\hline 1 & 0 & 0 & $(1,1)$ & $(1,-2)$ & $(2,-1)$ \\
\hline 1 & 0 & 1 & $(7,4)$ & $(4,-11)$ & $(11,-7)$ \\
\hline 1 & 0 & 1 & $(13,7)$ & $(7,-20)$ & $(20,-13)$ \\
\hline 1 & 0 & 1 & $(5,2)$ & $(2,-7)$ & $(7,-5)$ \\
\hline 1 & 1 & 0 & $(4,1)$ & $(1,-5)$ & $(5,-4)$ \\
\hline 1 & 1 & 1 & $(10,1)$ & $(1,-11)$ & $(11,-10)$ \\
\hline 1 & 1 & 2 & $(29,20)$ & $(20,-49)$ & $(49,-29)$ \\
\hline 1 & 1 & 3 & $(73,13)$ & $(13,-86)$ & $(86,-73)$ \\
\hline 1 & 2 & 0 & $(4,7)$ & $(7,-11)$ & $(11,-4)$ \\
\hline 1 & 2 & 2 & $(712,379)$ & $(379,-1091)$ & $(1091,-712)$ \\
\hline
\end{tabular}


In Table 1, one line represents a class of six solutions (cf. $\S 1$ ). In the column for $\pm(x, y)$ the three possible values for $(x, y)$ with $x \geq 0$ are printed.

\section{APPENDIX}

The following theorem is a corollary to a recent theorem of K. R. Yu (cf. [9]).

Theorem. Let $\alpha_{1}, \ldots, \alpha_{n}(n \geq 2)$ be nonzero algebraic numbers, $K=$ $\mathbb{Q}\left(\alpha_{1}, \ldots, \alpha_{n}\right),[K: \mathbb{Q}]=d$, and let $p$ be a rational prime number. As usual, let $i$ satisfy $i^{2}=-1$, and put $\omega=(-1+i \sqrt{3}) / 2$. Put

$$
(D, \delta)= \begin{cases}(d, 1) & \text { if }(p>2 \text { and } i \in K) \text { or }(p=2 \text { and } \omega \in K), \\ (2 d, 2) & \text { otherwise. }\end{cases}
$$

Let $\wp$ be a prime ideal of the ring of integers of $K$ lying above $p$, and let $f_{\wp}$ be its residue class degree. For $\alpha \in K, \alpha \neq 0$, we denote by $\operatorname{ord}_{\wp}(\alpha)$ the order to which $\wp$ divides the fractional ideal $(\alpha)$ generated by $\alpha$, and we set $\operatorname{ord}_{\wp}(0)=\infty$. Also, for $\alpha \neq 0$ we denote by $h(\alpha)$ the absolute logarithmic height of $\alpha$ (see, e.g., [5, §2] or [4, Appendix II]), and we put $\log \alpha=\log |\alpha|+i \operatorname{Arg} \alpha$, with $-\pi<\operatorname{Arg} \alpha \leq \pi$. For any $j \in\{1, \ldots, n\}$ we put

$$
V_{j} \geq \max \left\{h\left(\alpha_{j}\right), \frac{\left|\log \alpha_{j}\right|}{2 \pi D}, \frac{f_{\wp} \log p}{d}\right\},
$$

and $V=\max _{1 \leq j \leq n} V_{j}$. Let $b_{1}, \ldots, b_{n} \in \mathbb{Z}$, and $B=\max \left\{\left|b_{1}\right|, \ldots,\left|b_{n}\right|\right\}$. If $\operatorname{ord}_{\wp}\left(\alpha_{j}\right)=0$ for all $j=1, \ldots, n$ and $\alpha_{1}^{b_{1}} \cdots \cdots \alpha_{n}^{b_{n}} \neq 1$, then

$$
\operatorname{ord}_{\wp}\left(\alpha_{1}^{b_{1}} \cdots \cdots \alpha_{n}^{b_{n}}-1\right)<C_{1} C_{2} C_{3}\left(\log B+C_{4}\right) \text {, }
$$

where

$$
\begin{aligned}
& C_{1}= \begin{cases}35009 \cdot(45 / 2)^{n} & \text { if } p \equiv 1(\bmod 4), \\
30760 \cdot 25^{n} & \text { if } p \equiv 3(\bmod 4), \\
197142 \cdot 36^{n} & \text { if } p=2,\end{cases} \\
& C_{2}=(n+1)^{2 n+4} p^{\delta f_{p}}\left(f_{\wp} \log p\right)^{-(n+1)} D^{n+2} V_{1} \cdots V_{n}, \\
& C_{3}= \begin{cases}\log \left(2^{11}(n+1)^{2} D^{2} V\right) & \text { if } p>2, \\
\log \left(3 \cdot 2^{10}(n+1)^{2} D^{2} V\right) & \text { if } p=2,\end{cases} \\
& C_{4}=2 \log D .
\end{aligned}
$$

We can apply this theorem to the right-hand side of (8) as follows. Suppose that $n_{1} \geq 1$. Then

$$
\operatorname{ord}_{\wp_{17}}\left((-1)^{a_{2}} \xi^{n_{1}} \chi_{h}^{n_{2}} \vartheta^{a_{1}^{\prime}} \eta^{a_{2}^{\prime}}+1\right)=0
$$


and consequently

$$
n_{1}=\operatorname{ord}_{\wp_{17}}\left(\xi^{2 n_{1}} \chi_{h}^{2 n_{2}} \vartheta^{2 a_{1}^{\prime}} \eta^{2 a_{2}^{\prime}}-1\right)
$$

Analogously, we can do this with (9). Then we may apply the theorem with $B=2 H, D=6, \delta=2$, and $n=4$. Thus, upper bounds for $n_{1}$ and $n_{2}$ can easily be found by means of this theorem as follows:

$$
\begin{gathered}
\frac{f_{\wp} \log p}{d}<\left\{\begin{array}{l}
0.944405 \text { if } p=17, \\
0.981480 \text { if } p=19,
\end{array}\right. \\
h(\xi)<1.410173, \frac{|\log \xi|}{2 \pi D}<0.0371, \quad \text { hence } V_{\xi}=1.410173, \\
h(\chi)<1.381873, \frac{|\log \chi|}{2 \pi D}<0.032, \quad \text { hence } V_{\chi}=1.381873, \\
h(\vartheta)<0.352526, \frac{|\log \vartheta|}{2 \pi D}<0.088, \quad \text { hence } V_{\vartheta}= \begin{cases}0.944405 & \text { if } p=17, \\
0.981480 & \text { if } p=19,\end{cases} \\
h(\eta)<0.352526, \frac{|\log \eta|}{2 \pi D}<0.085, \\
\text { hence } V_{\eta}= \begin{cases}0.944405 & \text { if } p=17, \\
0.981480 & \text { if } p=19 .\end{cases}
\end{gathered}
$$

Here, of course, $V_{\alpha_{i}}$ stands for $V_{i}$. From these values we computed

$$
\begin{gathered}
C_{1}=\left\{\begin{array}{ll}
8.972424 \cdot 10^{9} & \text { if } p=17, \\
1.2015625 \cdot 10^{10} & \text { if } p=19,
\end{array} \quad C_{2}= \begin{cases}3.134053 \cdot 10^{13} & \text { if } p=17, \\
3.487752 \cdot 10^{13} & \text { if } p=19,\end{cases} \right. \\
C_{3}=14.77073,
\end{gathered}
$$

It follows that we can take for $c_{13}$ and $c_{14}$ (cf. (10)):

$$
c_{13}=6.190047 \cdot 10^{24}>C_{1} C_{2} C_{3}, \quad c_{14}=4.28>2 \log 6+\log 2 .
$$

Note that (10) is true also for $N=0$.

Addendum. After we had completed the present work, the following important papers appeared:

J. Blass, A. M. W. Glass, D. K. Manski, D. B. Meronk, and R. P. Steiner, Constants for lower bounds for linear forms in the logarithms of algebraic numbers II. The homogeneous rational case, Acta Arith. 55 (1990), 15-22.

Kunrui $\mathrm{Yu}$, Linear forms in p-adic logarithms. II, Compositio Math. 74 (1990), 15-113 (this is the paper announced in [9]).

The results included in these papers improve on the results for $c_{7}$ and $c_{13}$, respectively, and thus would have given an upper bound for $H$ that is considerably better than (19), although it still would have been very large. This would not have implied anything for our method, but it would have considerably reduced the computation time. 


\section{BIBLIOGRAPHY}

1. A. K. Agrawal, J. H. Coates, D. C. Hunt, and A. J. van der Poorten, Elliptic curves of conductor 11, Math. Comp. 35 (1980), 991-1002.

2. A. K. Lenstra, H. W. Lenstra Jr., and L. Lovász, Factoring polynomials with rational coefficients, Math. Ann. 261 (1982), 515-534.

3. T. N. Shorey and R. Tijdeman, Exponential diophantine equations, Cambridge Univ. Press, Cambridge, 1986.

4. N. Tzanakis and B. M. M. de Weger, On the practical solution of the Thue equation, J. Number Theory 31 (1989), 99-132.

5. M. Waldschmidt, $A$ lower bound for linear forms in logarithms, Acta Arith. 37 (1980), 257283.

6. B. M. M. de Weger, On the practical solution of Thue-Mahler equations, an outline, in: $\mathrm{K}$. Györy and G. Halász, Number Theory, Vol. II. Diophantine and Algebraic, Colloq. Math. Soc. J. Bolyai, Vol. 51, North-Holland, Amsterdam, Oxford, and New York, 1990, pp. 1037-1050.

7. __ Algorithms for diophantine equations, CWI-Tract No. 65, Centre for Mathematics and Computer Science, Amsterdam, 1989.

8. Kunrui Yu, Linear forms in p-adic logarithms, Acta Arith. 53 (1989), 107-186.

9. ___ Linear forms in p-adic logarithms. II (abstract), private communication.

Department of Mathematics, University of Crete, Iraklion, Greece

E-mail address: tzanakis@grearn.bitnet

Faculty of Applied Mathematics, University of Twente, P.O. Box 217, 7500 AE ENSCHEDE, THE NETHERLANDS

E-mail address: deweger@henut5.bitnet 